\title{
As dimensões do ser humano e o cuidado de enfermagem no contexto pandêmico da COVID-19
}

The dimensions of the human being and nursing care in the pandemic context of COVID-19

Las dimensiones del cuidado del ser humano y de la enfermería en el contexto pandémico de COVID-19

Paulo Henrique Alexandre de Paula ${ }^{1,2}$ (1)

Patricia Neyva da Costa Pinheiro 2 (B)

Edna Johana Mondragón-Sánchez $z^{2,3}$ (D)

Maria Isabelly Fernandes da $\operatorname{Costa}^{2}$ (1) Icleia Parente Rodrigues ${ }^{4}$ (D) João Víctor Lira Dourado ${ }^{5}$ (1)

1. Centro Universitário Inta. Sobral, Ceará, Brasil.

2. Universidade Federal do Ceará, Programa de Pós-graduação em Enfermagem. Fortaleza, Ceará, Brasil.

3. Universidad del Quindio, Programa de Enfermagem. Armenia, Colombia

4. Maternidade Escola Assis Chateaubriand. Fortaleza, Ceará, Brasil.

5. Universidade Federal do Ceará, Programa de Pós-graduação em Saúde Pública. Fortaleza, Ceará, Brasil.

Autor Correspondente:

Edna Johana Mondragón Sánchez

E-mail: ejmondragon@uniquindio.edu.com

Recebido em 05/08/2020.

Aprovado em 24/10/2020.

DOl:https://doi.org/10.1590/2177-9465-EAN-2020-0321

\section{RESUMO}

Objetivo: refletir sobre as dimensões do ser humano e o cuidado de enfermagem no contexto pandêmico da Covid-19. Método: trata-se de um estudo reflexivo, em que foram discutidas as dimensões corpórea (Homo somaticus), de vida humana (Homo vivens), conhecer (Homo sapiens), vontade (Homo volens), linguagem (Homo loquens), social (Homo socialis), do trabalho (Homo faber), jogo e divertimento (Homo ludens) e o cuidado de enfermagem no contexto pandêmico da Covid-19. Resultados: as dimensões estão sendo influenciadas pelo contexto pandêmico do novo coronavírus, sendo necessárias intervenções de enfermagem humanizadoras como o manejo clínico e terapêutico transpessoal, suporte ao corpo físico em adoecimento, garantia de uma linguagem que possibilite o autocuidado e apoio psicológico, atitude de escuta aberta, incentivo às práticas de jogos para passar o tempo e métodos de relaxamento, meditação e imaginação guiada, entre outras. $\mathrm{O}$ ato de refletir sobre as dimensões cria novas possibilidades de sinalizar pontos de intercessão para a compreensão da nova realidade vivenciada pelo homem. Conclusão e implicações para a prática: ao refletir sobre as dimensões do homem à luz da antropologia com o embasamento teórico dos Modelos de cuidados da Nursing Interventions Classification (NIC), geram-se possibilidades de estabelecer um cuidado holístico no contexto da pandemia pelo novo coronavírus.

Palavras-chave: Antropologia; Infecções por Coronavírus; Existencialismo; Cuidados de Enfermagem; Pandemias.

\section{ABSTRACT}

Objective: to reflect on the dimensions of the human being and nursing care in the pandemic context of Covid-19. Method: this is a reflection study, in which the corporeal dimensions (Homo somaticus), human life (Homo vivens), knowing (Homo sapiens), will (Homo volens), language (Homo loquens), social (Homo socialis), work (Homo faber), play and fun (Homo ludens) and nursing care in the pandemic context of Covid-19. Results: the dimensions are being influenced by the pandemic context of the new coronavirus, requiring humanizing nursing interventions, such as transpersonal clinical and therapeutic management, support for the sick physical body, guarantee of a language that enables self-care and psychological support, attitude of open listening, encouraging game practices to spend time and methods of relaxation, meditation and guided imagination, among others. The act of reflecting on the dimensions creates new possibilities for signaling points of intercession for the understanding of the new reality experienced by man. Conclusion and implications for practice: when reflecting on the dimensions of man in the light of anthropology with the theoretical basis of the Nursing Interventions Classification (NIC) Care Models, possibilities for holistic care are created in the context of the new coronavirus pandemic.

Keywords: Anthropology; Coronavirus Infections; Existentialism; Nursing Care; Pandemics.

\section{RESUMEN}

Objetivo: reflexionar sobre las dimensiones del ser humano y la atención de enfermería en el contexto pandémico de Covid-19. Método: este es un estudio reflexivo, en el que las dimensiones corporales (Homo somaticus), la vida humana (Homo vivens), el conocimiento (Homo sapiens), la voluntad (Homo volens), el lenguaje (Homo loquens), social (Homo socialis), trabajo (Homo faber), juego y diversión (Homo ludens) y cuidados de enfermería en el contexto pandémico de Covid-19. Resultados: las dimensiones están siendo influenciadas por el contexto pandémico del nuevo coronavirus, que requiere intervenciones humanizadoras de enfermería, como el manejo clínico y terapéutico transpersonal, el apoyo al cuerpo físico enfermo, la garantía de un lenguaje que permita el autocuidado y el apoyo psicológico, la actitud de escucha abierta, fomentando prácticas de juego para pasar el tiempo y métodos de relajación, meditación e imaginación guiada, entre otros. El acto de reflexionar sobre las dimensiones crea nuevas posibilidades para señalar puntos de intercesión para comprender la nueva realidad experimentada por el hombre. Conclusión e implicaciones para la práctica: reflexionar sobre las dimensiones del hombre a la luz de la antropología con la base teórica de los Modelos de Atención de la Clasificación de Intervenciones de Enfermería (NIC) genera posibilidades de atención integral en el contexto de la nueva pandemia de coronavirus.

Palabras clave: Antropología; Infecciones por Coronavirus; Existencialismo; Atención de Enfermería; Pandemias. 


\section{INTRODUÇÃO}

A Covid-19 é uma doença que apresenta um quadro clínico que varia de infecções assintomáticas a quadros respiratórios graves. De acordo com a Orgnização Mundial da Saúde, $80 \%$ dos pacientes podem ser assintomáticos e $20 \%$ dos casos podem requerer atendimento hospitalar pela presença de dificuldade respiratória. Desses casos, aproximadamente 5\% podem necessitar de suporte para o tratamento de insuficiência respiratória (suporte ventilatório)..$^{1,2}$ Declarada uma doença de caráter pandêmico, a infecção tornou-se uma emergência de Saúde Pública, atingindo o número de 35.179 .573 casos e 1.037.340 mortes, dados esses divulgados até cinco de outubro de $2020 .^{3}$

Todo esse contexto de enfretamento da infecção viral pelo SARS-COV-2 demandou alterações nas atividades de vida diárias do ser humano, afetando, assim, as dimensões da sua existência. Diante dessas alterações, destacam-se as repercussões significativas no processo saúde-doença que afetam o cuidado em saúde, em especial, àquelas relacionadas à prática da enfermagem pelo fato de suas habilidades e competências estarem relacionadas ao desenvolvimento de cuidados que vão desde a promoção da saúde e prevenção de agravos até a assistência de pacientes críticos de alta complexidade., ${ }^{4,5}$

A prática da enfermagem traz importantes contribuições na perspectiva da saúde do indivíduo, no sentido de reconhecer o ser humano como um ser complexo composto por diversas dimensões que vão do orgânico ao espiritual, do tangível ao intangível, incluindo o aspecto social e histórico, que o define como um ser de inter-relações permanentes consigo mesmo e com o mundo. . $^{6}$

Sob essa perspectiva, o humano é visto como detentor de características e atributos inerentes ao seu mundo-vida, destacando-se a consciência, o intelecto, a dignidade, as emoções, os sentimentos e o conhecimento. Portanto, o cuidado de enfermagem assume uma dimensão humanística e abrangente, cuja subjetividade é estruturada por suas experiências, significados, intuições e raciocínio. ${ }^{6,7}$

Dentro dessa visão humanística do cuidado, destaca-se a Teoria do Cuidado Transpessoal de Jean Watson que propõe um cuidado transcendental, capaz de contemplar o indivíduo em sua integralidade de corpo-mente-espírito. Essa filosofia de cuidado transcende a periferia de uma perspectiva de cuidado tradicional e alcança as profundezas da consciência dos sujeitos que participam do momento de cuidado. Portanto, o enfermeiro precisa abordar o mundo do outro para entendêlo e fortalecê-lo em sua própria capacidade de cuidar-se. Para tanto, esse profissional deve reconhecer a importância da sua própria existência, especialmente nesse cenário de crise pandêmica, em que suas habilidades têm se tornado relevantes para desempenhar o cuidado humano. ${ }^{6,7}$

Nessa perspectiva, a fenomenologia do homem permite a compreensão das várias dimensões humanas, desde a vida como conhecimento até a realidade funcional do corpo e como estas podem sofrer adaptações perante a pandemia. ${ }^{8}$ Diante da complexidade do ser humano e das influências vividas nos tempos atuais, são inquestionáveis as variadas consequências na vida das pessoas, causando mudanças no seu cotidiano. Portanto, buscou-se refletir sobre as dimensões humanas e sua importância para os cuidados de enfermagem na pandemia da Covid-19.

\section{MÉTODO}

Trata-se de um estudo de reflexão fundamentado nas dimensões do homem, embasadas na antropologia de Mondin, voltado para o cuidado de enfermagem, bem como na leitura crítica de estudos científicos atuais sobre a Covid-19. Esse tipo de pesquisa se aproxima da abordagem qualitativa, dada a interpretação e análise dos elementos teóricos obtidos por meio da investigação bibliográfica consultada. ${ }^{9}$

Apresentaram-se assuntos no campo das dimensões do homem no contexto pandêmico da Covid-19, conforme as dez dimensões: dimensão corpórea (Homo somaticus), a vida humana (Homo vivens), o conhecer sensitivo e intelectivo (Homo sapiens), a vontade, liberdade e amor (Homo volens), a linguagem (Homo loquens), a dimensão social (Homo socialis), a cultura (Homo culturalis), a do trabalho (Homo faber), o jogo e o divertimento (Homo ludens) e a religiosa (Homo religiosus). ${ }^{9}$

O estudo discute sobre as dimensões e as influências do contexto que podem levar às medidas de prevenção e à promoção da saúde do homem. Nessa perspectiva, a enfermagem apresenta destaque na busca de um cuidado que possibilite um conjunto de práticas, tecnologias e procedimentos voltados para a atenção à saúde e que transcenda o cuidado fisiológico e alcance as dimensões afetadas, como destacado na Teoria Transpessoal de Jean Watson e Teoria da diversidade e universalidade do cuidado cultural de Leininger, utilizadas nesta pesquisa para estabelecer reflexões sobre cuidados humanizados de enfermagem nas pessoas, famílias e comunidades.

\section{RESULTADOS E DISCUSSÃO}

\section{Cuidados de enfermagem e as dimensões do corpo, da vida, do saber e da vontade}

A expressão 'homo somaticus' faz alusão ao corpo, sob o prisma das propriedades relacionadas ao seu mecanismo em perfeito funcionamento tanto no conjunto quanto em suas partes singulares, além de suas funções de corporeidade, uma vez que é mediante o corpo que o homem é um ser social, é um ser no mundo. Para tanto, as principais funções da corporeidade, em geral, relacionam-se com as funções de mundanização, de individuação, de autocompreensão, de posse, entre outras. ${ }^{8}$

O corpo se sapresenta no contexto da Covid-19 como àquele com possibilidades de ser atingido pela doença, o que põe em risco suas funções fisiológicas sobretudo devido às alterações pulmonares provocadas pela doença. ${ }^{10} \mathrm{O}$ corpo, em sua realidade física, ao ser atingido por essa doença, apresenta alterações das suas propriedades. ${ }^{8}$

Ao se dar conta do corpo e da experiência da vida, o homem demonstra outra dimensão que é o saber. Isso acontece, pois ele é dotado de conhecimento sensitivo e intelectivo, tornando-o 
consciente do mundo ao seu redor. ${ }^{8}$ No entanto, o conhecimento da realidade pandêmica por meio do saber, mostra-se, por vezes, limitado. ${ }^{11}$

Outrossim, é possível refletir sobre a dimensão da vontade, caracterizada pelo dinamismo, movimento, atitudes e atividades do ser humano. O homem não tem poupado esforços, motivado por sua vontade, para combater a Covid-19 de forma individual ou coletiva, reunindo ações de promoção da sua saúde e bem-estar. ${ }^{11,12}$

O cuidado de enfermagem, em busca do alcance da saúde integral da pessoa, família e sociedade, nessa dimensão, relaciona-se com a vertente de sinais e sintomas marcado pela febre, cansaço, tosse seca e dificuldade para respirar. Essas alterações hemodinâmicas possuem níveis de gravidade que, indiscutivelmente, podem acarretar óbitos, refletindo na vida do indivíduo não só durante a doença, mas em todo o processo que ela percorre, transpondo o conhecimento técnico do diagnóstico e suas medidas de promoção, proteção e recuperação, até limitações de vida diária, como medidas de isolamento social, interferindo no domínio da vontade e na decisão do indivíduo. ${ }^{13,14}$

Nesse sentido, medidas de isolamento, higienização, identificação precoce dos sinais e sintomas sugestivos da infecção, seguimento e acompanhamento do tratamento medicamentoso, dentre outras, são consideradas estratégias fundamentais para o combate ao novo coronavírus. ${ }^{13}$

Entretanto, as pessoas não estão imunes à doença, e o acometimento pelo vírus revela uma experiência desafiadora durante o processo de hospitalização marcada pelo medo, ansiedade, preocupação com o desconhecido, valorização da opinião do paciente e transformação de práticas e atitudes profissionais. É o caso de idosos infectados pela COVID-19 na Espanha que tiveram a experiência de enfrentar desde os sintomas físicos da doença até os aspectos emocionais e sociais, reforçando a ideia da fragilidade da vida. A equipe, especialmente os enfermeiros, adotaram estratégias transformadoras como a comunicação pelo toque ou sons, já que estavam utilizando barreiras de proteção que dificultavam uma comunicação eficaz, e o compartilhamento de informações com os familiares por meio de ligações telefônicas para possibilitar o mais alto padrão possível de atendimento. ${ }^{15}$

A enfermagem oferece um cuidado integral, garantindo a saúde plena com resgate da autonomia, individualidade e integridade da vida de cada indivíduo, rementendo-se assim à Teoria Transpessoal de Jean Watson composta de dez elementos que consideram o cliente como o sagrado e propõe uma filosofia e ciência centradas no cuidado, o que constitui um dos principais eixos da prática de enfermagem (Quadro 1).

\section{Cuidados de Enfermagem e as dimensões da linguagem, do social e da cultura}

O homem, enquanto ser social que necessita se comunicar, utiliza a linguagem como seu maior instrumento. A linguagem pressupõe três condições transcendentais: o sujeito que fala, o objeto de que se fala e o interlocutor com quem quer se comunicar falando. ${ }^{8}$ Sendo assim, a linguagem revelada na atualidade reflete a preocupação do homem, e a comunidade científica se esforça para compreender os mecanismos que levam à doença, para que se possibilite por meio de uma linguagem clara, os devidos direcionamentos orientadores da sociedade na perspectiva da compreensão e adoção de medidas preventivas, além das formas de lidar com uma condição tão singular vivenciada no contexto atual. ${ }^{12-17}$

Quadro 1. Intervenções para o Cuidado Humanizado de Enfermagem para dimensões de corpo, vida, saber e vontade em tempos de Pandemia. Fortaleza, Brasil, 2020.

\begin{tabular}{l} 
Cuidados de Enfermagem Humanizados \\
\hline Pessoa, Família e Comunidade \\
- Atentar para o saber dos sinais e sintomas envolvidos, bem como atitudes a serem tomadas perante as identificações de \\
riscos; \\
- Incluir medidas preventivas que diminuam a disseminação do vírus na vida diária; \\
- Utilizar medidas de superação de enfrentamento de medos, angústias e incertezas perante a preservação da qualidade de \\
vida e saúde; \\
- Enfrentamento familiar eficaz para adição de medidas de suporte saudáveis e colaborativas; \\
- Decisão pessoal, individual e intransferível em ser participante da vontade de contribuir na diminuição da disseminação da \\
carga viral. \\
Profissionais de saúde \\
- Conhecer a pandemia, com destaque para identificação dos sinais e sintomas prevalentes, bem como sua condução \\
terapêutica; \\
- Atentar para o aumento da temperatura corporal; \\
- Alerta para sinais e sintomas de Síndrome Gripal; \\
- Manter padrão respiratório eficaz de vias áreas; \\
- Oferecer ao cliente segurança sobre a terapêutica desenvolvida;
\end{tabular}

Fonte: Elaboração baseada em Nursing Interventions Classification (NIC). ${ }^{16}$ 
O homem é essencialmente sociável, sozinho não teria condições de vir ao mundo nem realizar as suas aspirações mais elevadas. Para obtenção de todos esses anseios, faz-se necessária a companhia dos outros, cujas relações constituem a dimensão social, a qual está bastante comprometida devido às restrições impostas pelo distanciamento social.2,12,17

O comprometimento da dimensão social é expresso em relatos de pacientes que contraíram o novo coronavírus e precisaram de hospitalização ${ }^{18}$ ou ficaram em locais de quarentena, ${ }^{19}$ revelando o medo inicial diante da nova realidade, tendo em vista a quebra das relações sociais, mesmo considerando o isolamento social como necessário para proteger as demais pessoas, sensação de ser estigmatizado, para quem teve contato com pessoas com a doença e o apoio recebido de amigos e demais familiares.

$O$ apoio de enfermeiros tem-se revelado bastante significativo para o cuidado de pacientes com a Covid-19, independente de sua especialidade, em meio aos novos desafios de adquirir novos conhecimentos, cumprir novos protocolos e melhorar a qualidade física e psicológica para possibilitar o cuidado aos pacientes. ${ }^{20}$ Embora haja um intenso trabalho dos enfermeiros na linha de frente do cuidado, tanto física quanto emocionalmente, eles demonstram espírito de dedicação e se sentem responsáveis por colaborar com a vida dos pacientes em meio a pandemia. ${ }^{21}$

As pessoas socialmente isoladas, com mobilidade restrita e pouco contato com outras pessoas têm apresentado maior vulnerabilidade a complicações psiquiátricas que podem variar desde a insônia, ansiedade, depressão até transtorno de estresse pós-traumático (TEPT). ${ }^{22}$ Nesse contexto, a enfermagem apresenta-se atuante na assistência humanizada que transcende a assistência física, como sugere a teoria de Jean Watson de que, em um nível mais avançado do cuidado, a enfermagem é capaz de acessar os aspectos emocionais e subjetivos, de forma a objetivar a transpessoalidade por meio da comunicação e da empatia. ${ }^{8}$
Estes são os principais instrumentos para estabelecer e manter a relação de ajuda e confiança entre profissional e paciente, visto que o verdadeiro cuidar é pautado por uma relação empática, em que se reconhece o outro com quem vivencia sua experiência única de ser paciente. ${ }^{8}$

Outra dimensão importante que se revela em meio a esse cenário é a cultura na qual os modos de viver, comportamentos e hábitos são partes integrantes de cada indivíduo e influenciam sua saúde, como aponta Leininger em sua teoria da diversidade e universalidade do cuidado cultural (TDUCC), que propõe o cuidado sob a ótica transcultural e holística. ${ }^{23}$

O profissional deve dispor de uma postura empática, o que proporciona um equilíbrio tanto com o ambiente quanto com os pacientes. Esse comportamento ocasiona mudanças em um contexto desfavorável, atendendo às necessidades físicas, mentais e emocionais. ${ }^{8,23,24}$ Nesse sentido, é importante compreender as relações socioculturais no processo saúde e doença, ampliando o cuidado para além da dimensão biológica. ${ }^{22}$

Assim, o enfermeiro como um educador tem um papel fundamental na sensibilização da população quanto à adoção de hábitos que reduzam a disseminação da Covid-19, como permanecer em isolamento social, ter hábitos de higiene, lavar as mãos com frequência, higienizar objetos e usar máscaras, considerando o ambiente e as limitações de cada indivíduo ${ }^{25}$ (Quadro 2).

\section{Cuidados de Enfermagem e as Dimensões do Trabalho, do Jogo e Religião}

O trabalho é uma atividade importante para o estudo do homem, assim como o conhecimento, a liberdade e a linguagem, tendo em vista que o indivíduo é essencialmente criador de formas e fazedor de obras. ${ }^{8} \mathrm{O}$ trabalho é definido como toda atividade material e espiritual que procura resultado útil. Além disso, agrega elementos importantes para a sua compreensão como o uso do corpo para transmitir energia, esforço e perseverança. ${ }^{8}$

Quadro 2. Intervenções para o Cuidado Humanizado de Enfermagem baseadas nas dimensões da linguagem, do social e da cultura em tempos de pandemia da Covid-19. Fortaleza, Brasil, 2020.

\begin{tabular}{|l|}
\hline \multicolumn{1}{|c|}{ Cuidados de Enfermagem Humanizados } \\
\hline Pessoa, Família e Comunidade \\
- Usar uma abordagem calma e tranquilizadora; \\
- Tentar compreender a perspectiva do paciente e família em relação à situação temida; \\
- Escutar o paciente e o familiar com atenção; \\
- Encorajar a expressão de sentimentos, percepções e medos; \\
- Determinar a adequação das redes sociais existentes; \\
Profissionais de saúde \\
- Identificar eventos estressores no ambiente de trabalho; \\
- Iniciar programas de promoção da saúde no local de trabalho; \\
- Identificar e tratar condições agudas no trabalho; \\
- Iniciar mudanças no ambiente para eliminar ou minimizar risco; \\
- Utilizar técnicas de relaxamento; \\
\hline
\end{tabular}

Fonte: Elaboração baseada em Nursing Interventions Classification (NIC). ${ }^{16}$ 
Entretanto, essa dimensão está significativamente afetada decorrente da recessão econômica global, tendo em vista os efeitos sobre o emprego das pessoas. Por isso, a ansiedade, a preocupação, o estresse, os problemas com sono e apetite, bem como a mistura de sentimentos ou emoções ficam presentes no povo, em virtude da incerteza da situação, assim como o impacto psicológico principalmente nos profissionais de saúde que estão na linha de frente, sendo o estresse uma constante em seu cotidiano.

A pandemia tem revelado uma nova configuração no ambiente de trabalho dos enfermeiros e reforçou com maior amplitude as condições precárias de trabalhos, renda, saúde física e mental destes profissionais, havendo a necessidade de estudos que reforcem essa realidade com o intuito de sensibilizar e propor sugestões às Entidades de Classe, à sociedade e ao Estado na perspectiva de um trabalho decente. ${ }^{26}$

Mesmo diante dos desafios apresentados, é válido refletir também sobre a dimensão relacionada ao jogo, divertimento e recreação, pois, diferentemente das apresentadas até aqui, essa dimensão pode ajudar a tirar o foco dos aspectos negativos da realidade vivenciada. O homem inventa jogos e se diverte como nenhum outro animal sabe fazer. Dessa forma, ele também é definido como ludens. O jogo apresenta propriedades específicas que são a distração e o divertimento, sendo assim qualquer atividade feita com o intuito de divertir e distrair forma parte da categoria dos jogos. ${ }^{8}$

É válido refletir sobre essa dimensão no contexto da Covid-19, uma vez que o jogo também pode ser uma possibilidade de ocupar o tempo nesse momento de isolamento social. ${ }^{27} \mathrm{~A}$ atenção à necessidade de recreação é, contudo, uma condição indispensável para o cuidado de enfermagem e deve estar mais presente no momento, pois é uma das estratégias utilizadas no isolamento social, além da existência do diagnóstico de enfermagem Deficit nas atividades recreativas, que permite realizar uma série de intervenções, justificando o conhecimento dessa questão pelos profissionais de enfermagem. ${ }^{4}$

Por fim, a dimensão religiosa exprime-se como uma manifestação típica e exclusivamente no homem. Os antropólogos destacam que o homem desenvolveu a atividade religiosa desde a sua primeira aparição na cena da história, com uma impressão em todas as culturas. Há diversas críticas de natureza filosófica, teológica e histórica em torno dessa dimensão que ajuda a compreender a sua importância sob diferentes ângulos. Entretanto, é válido considerar a definição da religião como o conjunto de conhecimentos de ações e de estruturas com que o homem exprime reconhecimento, dependência e veneração em relação ao Sagrado. ${ }^{8}$

Dada a reflexão em torno da dimensão religiosa, é provável que esta contribua, entre outros aspectos, para a saúde mental das pessoas. ${ }^{28}$ Ainda assim, os papéis da religiosidade e espiritualidade mostram-se mais aguçados nesse momento por propiciarem o fortalecimento para a vivência diária das dificuldades ${ }^{28,29} \mathrm{~A}$ religiosidade é um recurso para construir a esperança em vida, implicando na relação do homem com um ser transcendente. ${ }^{28}$ Essas reflexões são importantes para se pensar no fortalecimento dessa dimensão para ajudar as pessoas no enfrentamento das dificuldades.

Jean Watson tem em sua teoria do cuidado humanizado um fator chamado "Incorporação da fé - esperança", em que fala da importância da fé e da esperança no cuidado e na cura, uma vez que está presente em nossa história, como também em toda a narrativa que explica nossa coexistência como espécie humana, na qual houve influências de magia, orações e encantos (Quadro 3).

Quadro 3. Intervenções para o Cuidado Humanizado de Enfermagem para dimensões do Trabalho, Jogo e Religião em tempos de Pandemia. Fortaleza, Brasil, 2020.

\section{Cuidados de Enfermagem Humanizados}

\section{Trabalho}

\section{Pessoa, Família e Comunidade}

- A primeira coisa é manter uma atitude de escuta aberta ao que as pessoas manifestam;

- Avalie cuidadosamente as reações das pessoas afetadas e identifique, com critérios claros, as que requerem atenção psicológica mais urgente para oferecer suporte personalizado;

- Você precisa fazê-la sentir que está acompanhada, segurar a sua mão, abraçá-la, acariciá-la, falar com ela em um tom afetuoso e sereno, chamando-a pelo nome;

- Se a pessoa expressar seus sentimentos de dor ou frustração com comportamento agressivo, como bater em objetos, acompanhe-a com calma, sem tentar detê-los imediatamente, a menos que a segurança de si ou de outras pessoas estejam em risco;

\section{Profissionais de saúde}

- Passe algum tempo com os colegas e desabafe para verbalizar pensamentos de situações vividas;

- Evite críticas destrutivas que afetam a todos;

- Tente descansar a cada duas horas, respirando fundo por alguns minutos para ajudar a relaxar;

- Ao sair do trabalho, evite o excesso de informações sobre a pandemia (TV, rádio, internet e WhatsApp).

Fonte: Elaboração baseada em Nursing Interventions Classification (NIC). ${ }^{16}$ 
Quadro 3. Continuação...

\begin{tabular}{l} 
Cuidados de Enfermagem Humanizados \\
\hline Jogo \\
- Usando atividades relaxantes, interessantes e de lazer para promover o bem-estar; \\
- Uso da música para ajudar a alcançar uma mudança específica no comportamento, sentimentos ou fisiológicos; \\
- Facilitação da comunicação por meio de desenhos ou outras formas de arte; \\
- Proporcionar um ambiente calmo; \\
- Incentive a pessoa a descrever seus desenhos ou criações artísticas; \\
Religião \\
- Identifique as necessidades espirituais e religiosas dessa pessoa, usando o conhecimento para valorizar e apresentar \\
cuidados espirituais e religiosos diariamente; \\
- Proporcionar um ambiente que favoreça a pessoa com a possibilidade de uma atitude meditativa / contemplativa de \\
autorreflexão; \\
- Esteja aberto a ouvir ativamente expressões e preocupações de solidão e impotência em tempos de pandemia e, se \\
necessário, ajude o indivíduo a expressar e liberar sentimentos negativos de forma adequada; \\
- Use técnicas para ajudar o indivíduo a classificar suas crenças e valores, bem como suas áreas e motivos de esperança na \\
vida; \\
- Incentive a pessoa a se relacionar com familiares, amigos e outras pessoas;
\end{tabular}

Fonte: Elaboração baseada em Nursing Interventions Classification (NIC). ${ }^{16}$

\section{CONCLUSÃO E IMPLICAÇÕES PARA A PRÁTICA}

O entendimento das dimensões corpórea, vida humana, saber, vontade, linguagem, social, cultural, trabalho, jogo e religiosa dá suporte para o cuidar, oportunizando a refexão das distintas dimensões do homem, compreendendo àquelas que necessitam ser estimuladas e trabalhadas sob o cuidado de enfermagem para o equilíbrio necessário do corpo físico, social, emocional e espiritual, os quais não podem ser traduzidos ainda em números e métodos concretos para compreensão da realidade em meio à existência do ser humano durante essa realidade.

A construção dos modelos de cuidados baseados na Nursing Interventions Classification (NIC) e o embasamento teórico criam possibilidades para um cuidado holístico, auxiliando as pessoas a reforçarem as suas potencialidades e/ou minimizarem desequilíbrios em seu estado de saúde, em especial, em meio à pandemia da Covid-19, considerando desde a dimensão fisiológica e orgânica até a dimensão social e espiritual.

Acredita-se que este trabalho se torna relevante, uma vez que instrumentaliza o cuidado ao homem na perspectiva holística, em situações de isolamento social, adoecimento do corpo, impedimento para o trabalho ou risco ocupacional, bem como outros aspectos em meio às incertezas do contexto pandêmico.

A limitação do estudo é que ainda não se tem como avaliar na prática as considerações teóricas abordadas. Além disso, não se tem como saber quais das dimensões estão mais afetadas; mas que o estudo tem o potencial de direcionar os cuidados de enfermagem no contexto da Covid-19.

Porém, sugere-se que novas abordagens dessa natureza sejam implementadas, a fim de possibilitar uma discussão mais ampliada e intensa quanto aos aspectos que envolvem as dimensões do homem de forma geral e no contexto pandêmico da Covid-19.

\section{FINANCIAMENTO}

Bolsa concedida a Edna Johana Mondragón Sánchez. Programa de Excelência Acadêmica da Coordenação de Aperfeiçoamento de Pessoal do Nível Superior (CAPES/PROEX - Processo 88887.334871/2019-00).

\section{CONTRIBUIÇÕES DOS AUTORES}

Desenho do estudo de reflexão. Paulo Henrique Alexandre de Paula. Patricia Neyva da Costa Pinheiro. Edna Johana Mondragón-Sánchez.

Levantamento das informações para elaborar a reflexão. Patricia Neyva da Costa Pinheiro. Edna Johana MondragónSánchez. Icleia Parente Rodrigues. Maria Isabelly Fernandes da Costa. João Víctor Lira Dourado

Análise reflexiva. Patricia Neyva da Costa Pinheiro. Edna Johana Mondragón-Sánchez. Icleia Parente Rodrigues. Maria Isabelly Fernandes da Costa. João Víctor Lira Dourado

Interpretação reflexiva. Patricia Neyva da Costa Pinheiro. Icleia Parente Rodrigues. Maria Isabelly Fernandes da Costa. João Víctor Lira Dourado

Redação e revisão crítica do manuscrito. Paulo Henrique Alexandre de Paula. Patricia Neyva da Costa Pinheiro. Edna Johana Mondragón-Sánchez. Maria Isabelly Fernandes da Costa. Icleia Parente Rodrigues. João Víctor Lira Dourado 
Aprovação da versão final do artigo. Paulo Henrique Alexandre de Paula. Patricia Neyva da Costa Pinheiro. Edna Johana Mondragón-Sánchez. Maria Isabelly Fernandes da Costa. Icleia Parente Rodrigues. João Víctor Lira Dourado

Responsabilidade por todos os aspectos do conteúdo e a integridade do artigo publicado. Paulo Henrique Alexandre de Paula. Patricia Neyva da Costa Pinheiro. Edna Johana MondragónSánchez. Maria Isabelly Fernandes da Costa. Icleia Parente Rodrigues. João Víctor Lira Dourado

\section{EDITOR ASSOCIADO}

\author{
Cristina Rosa Baixinho
}

\section{REFERÊNCIAS}

1. Chaves TSS, Bellei N. "SARS-COV-2, the new coronavirus: a reflection about 'One Health' and the importance of travel medicine when new pathogens emerge. Revista de Medicina [Internet]. 2020 [citado 2020 jun 20];99(9):1-4. Disponível em: https://www.revistas.usp.br/revistadc/ article/view/167173/159662

2. Ministério da Saúde (BR). Sobre a doença [Internet]. Brasil: Ministério da Saúde; 2020 [citado 2020 jun 20]. Disponível em: https://coronavirus. saude.gov.br/sobre-a-doenca

3. Johns Hopkins University. Center for Systems Science and Engineering COVID-19 [Internet]. United States of America: Center for Systems Science and Engineering; 2020 [citado 2020 out 05]. Disponível em: https://gisanddata.maps.arcgis.com/apps/opsdashboard/index.html\#/ bda7594740fd40299423467b48e9ecf6

4. Hill B. Coronavirus: origins, signs, prevention and management of patients. Br J Nurs. [Internet] 2020 [citado 2020 out 05];9(29):399-402. Available from: https://www.magonlinelibrary.com/doi/full/10.12968/ bjon.2020.29.7.399

5. Organização Pan-Americana da Saúde. Perspectivas e contribuições da enfermagem para promover a saúde universal [Internet]. Brasília: Organização Pan-Americana da Saúde; 2020 [citado 2020 mai 27] Disponível em: https://iris.paho.org/handle/10665.2/52121

6. Watson Caring Science Institute. 10 Caritas Processes [Internet] Boulder, CO: Watson Caring Science Institute; 2019 [citado 2020 ma 06]. Disponível em: https://www.watsoncaringscience.org/jean-bio/ caring-science-theory

7. Margadant C, Wortel S, Hoogendoorn M, Bosman R, Spijkstra JJ, Brinkman $S$ et al. The Nursing Activities Score per nurse ratio is associated with in-hospital mortality, whereas the patients per nurse ratio is not. Crit. Care Med. 2020 jan;48(1):3-9. http://dx.doi.org/10.1097/ CCM.0000000000004005

8. Credland N. Critical care nurses will need our support as COVID-19 cases rise [Internet]. Nurs Stan. 2020 [citado 2020 june 20]. Available from: https://rcni.com/nursing-standard/opinion/comment/critical-carenurses-will-need-our-support-covid-19-cases-rise-158836

9. Mondin B. O homem, que é ele? Elementos de antropologia filosófica. São Paulo: Edições Paulinas; 1980.

10. Minayo MCS. O desafio do conhecimento: pesquisa qualitativa em saúde. 9. ed. São Paulo: EDUC; 2006.

11. Chena Z, Fan H, Cai J, LiY, Wu B, Hou Y et al. High-resolution computed tomography manifestations of COVID-19 infections in patients of different ages. Eur. J. Radiol. [Internet]. 2020 mai [cited 2020 june 20];126(1):108972. Available from: https://www.sciencedirect.com/ science/article/pii/S0720048X20301613

12. Ford N, Vitoria M, Rangaraj A, Norris SL, Calmy A, Doherty M. Systematic review of the efficacy and safety of antiretroviral drugs against SARS, MERS, or COVID-19: initial assessment. J. Int. Aids soc. [Internet]. 2020 abr [cited 2020 june 20];23(4): e25489. Available from: https://pubmed. ncbi.nlm.nih.gov/32293807/
13. Gennaro FD, Pizzol P, Marotta C, Antunes M, Racalbuto V, Veronese $\mathrm{N}$ et al. Coronavirus Diseases (COVID-19) current status and future perspectives: a narrative review. Int. J. Environ. Res. Public health (Online) [Internet]. 2020 abr [citado 2020 june 20]; 17(8):2690. Disponível em: https://www.mdpi.com/1660-4601/17/8/2690

14. Ministério da Saúde (BR). Secretaria de Atenção Primária à Saúde. Protocolo de Manejo Clínico do Coronavírus (COVID-19) na Atenção Primária à Saúde [Internet]. Brasília: Ministério da Saúde; 2020. [citado 2020 mai 7]. Disponível em: https://saude.rs.gov.br/upload/ arquivos/202004/14140606-4-ms-protocolomanejo-aps-ver07abril.pdf

15. Merodio G, Ramis-Salas M, Valero D, Aubert, A. How Much Is One Life Worth? The right to equity healthcare for improving older patients' health infected by COVID-19. Sustainability [Internet]. 2020 [citado 2020 out 6];12:6848. Disponível em: file:///C:/Users/user/Downloads/ sustainability-12-06848.pdf

16. Bulechek GM, Butcher HK, Dochterman JM. Classificação das Intervenções de Enfermagem - NIC. $5^{\underline{a}}$ ed. Rio de Janeiro: Elsevier 2010.

17. Costa JR, Arruda GO, Barreto MS, Serafim D, Sales CA, Marcon SS Cotidiano dos profissionais de enfermagem e Processo Clinical Caritas de Jean Watson: uma relação. Rev Enferm UERJ. 2019; 27:e37744. http://dx.doi.org/10.12957/reuerj.2019.37744

18. Carr KC. My Coronavirus Experience. Nurs. Womens Health. [Internet] 2020 [citado 2020 out 6];24(4):305-6. Disponível em: https://www. sciencedirect.com/science/article/pii/S1751485120300969?via\%3Dihub

19. Chen D, Song F, Tang L, Zhang H, Shao J, Qiu R et al. Quarantine experience of close contacts of COVID-19 patients in China: A qualitative descriptive study. Gen. Hosp. Psychiatry. [Internet]. 2020 set [citado 2020 out 6];66:81-88. Disponível em: https://www.sciencedirect.com/ science/article/pii/S0163834320301079

20. Fan J, Hu K, Li X, Jiang Y, Zhou X, Gou X et al. A qualitative study of the vocational and psychological perceptions and issues of transdisciplinary nurses during the COVID-19 outbreak. Aging. [Internet]. 2020 [citado 2020 out 08];12(13):12479-92. Disponível em: https://www.ncbi.nlm. nih.gov/pmc/articles/PMC7377893/

21. Liu YE, Zhai ZC, Han YH, Liu YL, Liu FP, Hu DY. Experiences of front-line nurses combating coronavirus disease-2019 in China: A qualitative analysis. Public Health Nurs. [Internet]. 2020 [citado 2020 out 6];37(5):757-63. Disponível em: https://onlinelibrary.wiley.com/doi/ epdf/10.1111/phn.12768

22. Drakuliü AM, Radman V. Crisis psychodrama in the era of covid-19 Psychiatr. Danub. [Internet]. 2020 [citado 2020 jun 20]; 32(1):22-24. Disponível em: http://www.psychiatria-danubina.com/UserDocsImages/ pdf/dnb_vol32_no1/dnb_vol32_no1_22.pdf

23. Leininger MM. Ethical and Moral Dimensions of Care. Detroit: Wayne State University Press; 1990.

24. Nascimento HG, Figueiredo AEB. Demência, familiares cuidadores e serviços de saúde: o cuidado de si e do outro. Cien Saude Colet [Internet]. 2019 [citado 2020 out 8]; 24(4):1381-92. Disponível em: https://www.scielo.br/pdf/csc/v24n4/1413-8123-csc-24-04-1381.pdf

25. Watson J. Watsons theory of human caring and subjective living experiences: carative factors/caritas processes as a disciplinary guide to the professional nursing practice. Texto \& contexto enferm. [Internet]. 2007 Mar [citado 2020 out 8]; 16(1):129-135. Disponíve em: http://www.scielo.br/scielo.php?script=sci_arttext\&pid=S010407072007000100016\&lng=en

26. Machado MH, Pereira EJ, Ximenes Neto FRG, Wermelinger MCDMW Enfermagem em tempos de COVID-19 no Brasil: um olhar da gestão do trabalho. Enferm. Foco (Brasília). [Internet]. 2020 [citado 2020 jun 20];11(1):32-39. Disponível em: file:///C:/Users/user/Downloads/399421245-1-PB.pdf

27. Güner R, Hasanoglu I, Aktas F. COVID-19: Prevention and control measures in community. Turk J Med Sci. 2020; 50:571-577.

28. Ommeren MV, Hanna F, Weissbecker I, Ventevogel P. Mental health and psychosocial support in humanitarian emergencies. East Mediterr health J.2015;21(7):498-502. http://dx.doi.org/10.26719/2015.21.7.498.

29. Silva TF, Almeida FS, Souza NP. Dançar e brincar: uma experiência de balé com crianças pequenas. Pensar Prát. 2019;22:1-12. http://dx.doi. org/10.5216/rpp.v22.50553. 